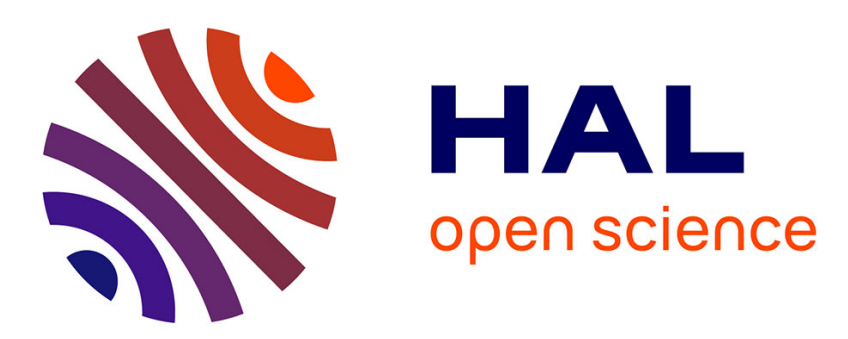

\title{
Xenon for tunnelling analysis of the efflux pump component OprN
}

\author{
Yvette Véronique Ntsogo Enguéné, Gilles Phan, Cyril Garnier, Arnaud \\ Ducruix, Thierry Prangé, Isabelle Broutin
}

\section{To cite this version:}

Yvette Véronique Ntsogo Enguéné, Gilles Phan, Cyril Garnier, Arnaud Ducruix, Thierry Prangé, et al.. Xenon for tunnelling analysis of the efflux pump component OprN. PLoS ONE, 2017. hal-02150038

\section{HAL Id: hal-02150038 \\ https://hal.science/hal-02150038}

Submitted on 6 Jun 2019

HAL is a multi-disciplinary open access archive for the deposit and dissemination of scientific research documents, whether they are published or not. The documents may come from teaching and research institutions in France or abroad, or from public or private research centers.
L'archive ouverte pluridisciplinaire HAL, est destinée au dépôt et à la diffusion de documents scientifiques de niveau recherche, publiés ou non, émanant des établissements d'enseignement et de recherche français ou étrangers, des laboratoires publics ou privés. 


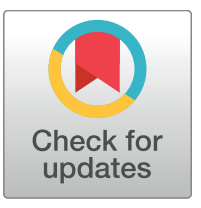

\section{G OPENACCESS}

Citation: Ntsogo Enguéné YV, Phan G, Garnier C, Ducruix A, Prangé T, Broutin I (2017) Xenon for tunnelling analysis of the efflux pump component OprN. PLoS ONE 12(9): e0184045. https://doi.org/ 10.1371/journal.pone.0184045

Editor: Eric Cascales, Centre National de la Recherche Scientifique, Aix-Marseille Université, FRANCE

\section{Received: April 28, 2017}

Accepted: August 17, 2017

Published: September 8, 2017

Copyright: @ 2017 Ntsogo Enguéné et al. This is an open access article distributed under the terms of the Creative Commons Attribution License, which permits unrestricted use, distribution, and reproduction in any medium, provided the original author and source are credited.

Data Availability Statement: All coordinate files are available from the PDB database (accession number(s) 5iuy, 5nsw).

Funding: This study was supported by the Agence Nationale de la Recherche (ANR-12-BSV8- 001001). YN was supported by both Vaincre la Mucoviscidose and the Grégory Lemarchal Associations.

Competing interests: The authors have declared that no competing interests exist.

\section{Xenon for tunnelling analysis of the efflux pump component OprN}

\author{
Yvette Véronique Ntsogo Enguéné ${ }^{1,2}$, Gilles Phan ${ }^{1 \oplus}$, Cyril Garnier ${ }^{1}$, Arnaud Ducruix ${ }^{1}$, \\ Thierry Prangé ${ }^{1 *}$, Isabelle Broutin ${ }^{1 *}$ \\ 1 Laboratoire de Cristallographie et RMN Biologiques (UMR 8015, CNRS), Faculté de Pharmacie, Université \\ Paris Descartes, USPC, Paris, France, 2 Laboratoire de Biologie Physico-Chimique des Protéines \\ Membranaires (UMR 7099, CNRS, Université Paris Diderot) Institut de Biologie Physico-Chimique, Paris, \\ France \\ จ These authors contributed equally to this work. \\ * isabelle.broutin@ parisdescartes.fr (IB); thierry.prange@ @ parisdescartes.fr (TP)
}

\section{Abstract}

Tripartite efflux pumps are among the main actors responsible for antibiotics resistance in Gram-negative bacteria. In the last two decades, structural studies gave crucial information about the assembly interfaces and the mechanistic motions. Thus rigidifying the assembly seems to be an interesting way to hamper the drug efflux. In this context, xenon is a suitable probe for checking whether small ligands could act as conformational lockers by targeting hydrophobic cavities. Here we focus on OprN, the outer membrane channel of the MexEF efflux pump from Pseudomonas aeruginosa. After exposing OprN crystals to xenon gas pressure, 14 binding sites were observed using $X$-ray crystallography. These binding sites were unambiguously characterized in hydrophobic cavities of OprN. The major site is observed in the sensitive iris-like region gating the channel at the periplasmic side, built by the three key-residues Leu 405, Asp 109, and Arg 412. This arrangement defines along the tunnel axis a strong hydrophobic/polar gradient able to enhance the passive efflux mechanism of OprN. The other xenon atoms reveal strategic hydrophobic regions of the channel scaffold to target, with the aim to freeze the dynamic movements responsible of the open/ close conformational equilibrium in OprN.

\section{Introduction}

Pseudomonas aeruginosa is an opportunistic bacterium frequently found in nosocomial infections and chronic lung infections of Cystic Fibrosis patients. Nowadays it is a serious challenge in hospital environments because of the appearance of multidrug resistant strains [1,2]. The intrinsic and acquired resistance phenotypes are highly linked to the rapid and active efflux of the drugs by a membrane protein complex made of three protein partners [3, 4], called the efflux pump system. In P. aeruginosa, at least 12 systems have been biochemically characterized [5], such as MexA-MexB-OprM (or commonly named MexAB-OprM) which is constitutively expressed, and MexE-MexF-OprN (or MexEF-OprN), which expression is triggered under antibiotic pressure [6, 7]. In MexAB-OprM, MexB belongs to the RND (Resistance 
Nodulation cell Division) membrane transporter superfamily, which actively pump out the drug at the inner-membrane; MexA is a periplasmic component (MFP or Membrane Fusion Protein) that stabilizes the whole system, and OprM is a tunnel-like component which allows the routing of the drug through the outer-membrane following a passive mechanism (Fig 1 left, for a review see [8]). Although a fully assemble efflux pump was recently described by single-particle electronic microscopy $[9,10]$, the selective export mechanism of the drug by outer-membrane channels remains elusive.

There is a large amount of data regarding the structure of the homotrimeric outer-membrane channel. Mutagenesis [11], X ray diffraction structures [12-14], normal mode analysis [13] and in silico dynamics [15], have converged toward a plausible mechanism of channel gating where an iris-like motion of the $\alpha$-helical coiled-coil domain [14] is combined with a twist/stretch mechanism [13]. Systematic mutagenesis on one hand, and salt-bridge analysis on the other hand, gave additional information on residues involved in the drugs efflux $[8,11]$. In addition, cobalt hexamine was found to inhibit the homologous channel TolC from E. Coli and the X-ray structure revealed that the inhibition took place at the conserved residues Asp 374 / Asp 371 [16]. Moreover, molecular dynamics simulations supported a special function of residues Val 408 and Asp 416 in OprM gating [15].

To gain more information regarding the dynamics of the OMF tunnelling mechanism, we focused our interest on OprN, the OMF protein associated with the MexEF complex within P. aeruginosa (Fig 1 right). This efflux pump is implicated in the resistance to fluoroquinolones, the most used antibiotic class. Although OprN has only a moderate degree of homology with OprM (31.4\%), its structure was solved by Molecular Replacement with OprM X-ray structure $[17,18]$. To determine whether small hydrophobic ligands could block the iris-like

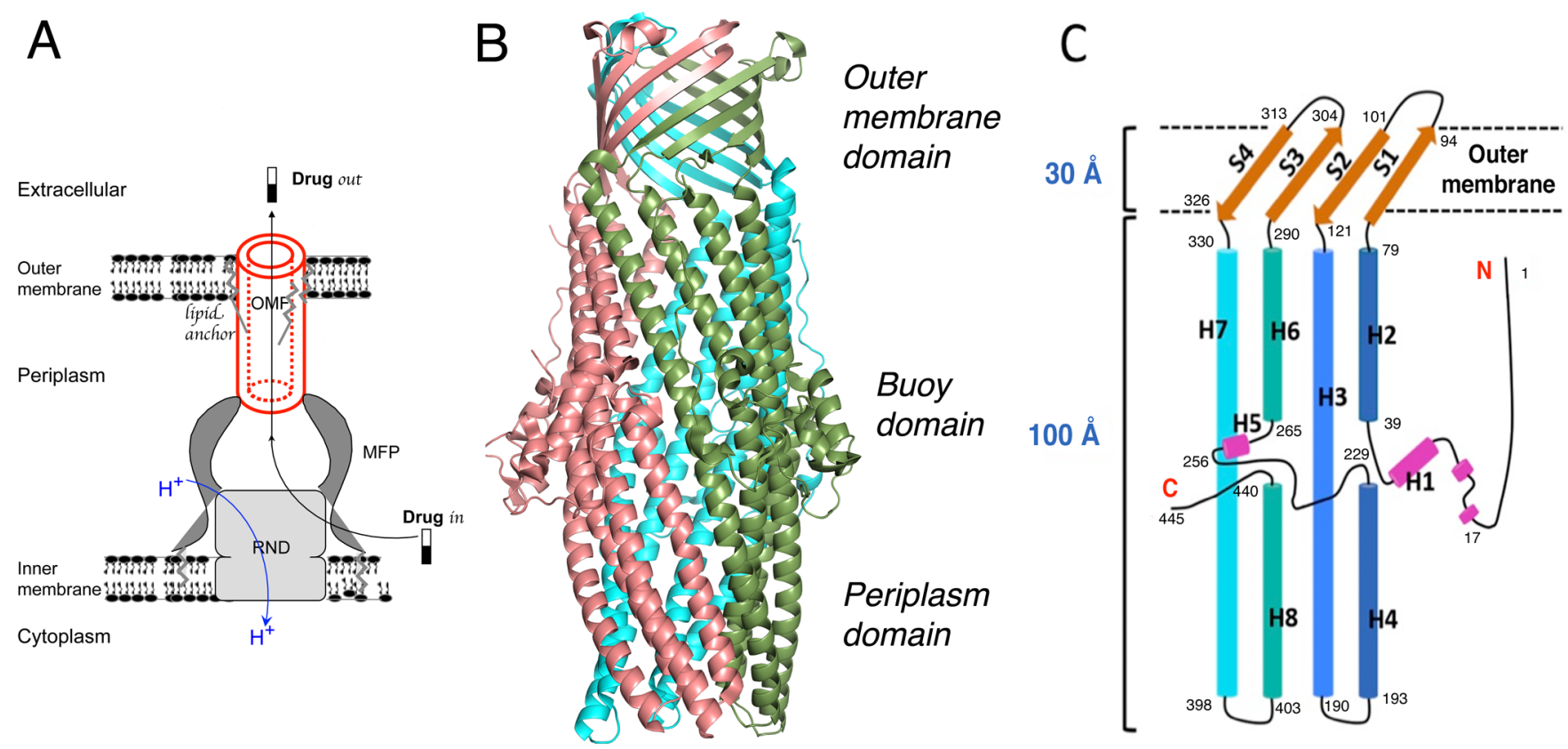

Fig 1. Views of the tripartite assembly of an efflux pump. A: The schematic reconstitution complex supported by microscopy images on the MexAB-OprM in nanodiscs and by the chimeric AcrAB-TolC-AcrZ complex [9]. The oligomerization state is 3:6:3 for the three components, respectively. The RND part uses the proton motive force and is responsible of the active counter transport of the drug. $\mathbf{B}$ : The overall trimer assembly of OprN (the OMF component -in red at left- of the MexEF-OprN assembly). C: Topology of secondary structures of the OprN monomer with $\beta$-strands $\mathrm{S} 1$ to $\mathrm{S} 4$ and $\alpha$-helices $\mathrm{H} 1$ to $\mathrm{H} 7$.

https://doi.org/10.1371/journal.pone.0184045.g001 
gate or freeze the channel conformation by targeting non-polar cavities, xenon appeared to be an ideal probe [19-23].

\section{Materials and methods OprN purification and crystallization}

The oprN gene was cloned into the plasmid pBAD-33 with a 6-His tag at the C-terminus. The purification protocol was already described [18] but some modifications were introduced as follows. After transformation in $\mathrm{C} 43-\Delta \mathrm{acrB}$ strain, the culture was performed at $37^{\circ} \mathrm{C}$ until an $\mathrm{OD}_{600}=0.6$, then cooled-down at $4^{\circ} \mathrm{C}$ during 30 minutes before induction with a final concentration of $0.02 \% \mathrm{~L}$-arabinose. Culture was continued overnight at $20^{\circ} \mathrm{C}$. The cell pellets were resuspended in $20 \mathrm{mM}$ Tris- $\mathrm{HCl} \mathrm{pH} \mathrm{8,150} \mathrm{mM} \mathrm{NaCl}$, lysed by two runs at $2.4 \mathrm{kbar}$ in a Celldisruptor (Constant Systems Ltd), and centrifuged for 30 minutes at $8000 \mathrm{~g}$. The supernatant was then centrifuged at $145000 \mathrm{~g}$ for 1 hour at $4^{\circ} \mathrm{C}$ to isolate the membranes. The pellet was dissolved in the same buffer with $0.5 \%(\mathrm{w}: \mathrm{w}) \mathrm{N}$-Lauryl-Sarcosine to specifically solubilize the inner membrane and centrifuged 1 hour at $145000 \mathrm{~g}$ leading to a pellet containing the outer membrane only. It was solubilized overnight at $4^{\circ} \mathrm{C}$ in the buffer with $1.5 \%$ (w:v) DDM (n-Dodecyl- $\beta$-D-Maltopyranoside) and the detergent was exchanged with $0.9 \%$ (w:v) $\beta$-OG

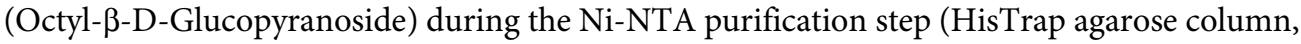
GE Healthcare). A last size exclusion chromatography step was performed on a Superose 6 column (GE Healthcare) in $20 \mathrm{mM}$ Tris- $\mathrm{HCl} \mathrm{pH} 8,150 \mathrm{mM} \mathrm{NaCl}$ and $0.9 \%$ (w:v) $\beta$-OG. The purified protein was concentrated to $12 \mathrm{mg} / \mathrm{mL}$ for crystallization. Needle crystals were obtained within a week at $18^{\circ} \mathrm{C}$ by mixing equal volumes of protein with $100 \mathrm{mM}$ sodium acetate, $\mathrm{pH} 5.4,0.8-1.1 \mathrm{M}$ sodium formate and 9-11\% PEG 4000 (w/v). Finally $30 \%$ glycerol was added to the crystallization drop as cryo-protectant before flash-cooling in liquid nitrogen.

\section{X-ray data collection and integration}

Diffraction data for OprN native structure (OprN-wt) were recorded at the ESRF synchrotron (Grenoble, France) on ID23-2 beamline at a wavelength of $0.873 \AA$, with a Pilatus3-2M detector (Dectris). Xenon bound derivative (OprN-Xe) crystals were obtained from OprN-wt crystals incubated with a pressure of $2 \mathrm{MPa}(20 \mathrm{bar})$ of xenon. After about 2-4 minutes, the pressure was released and the crystal immediately plunged in liquid nitrogen for quenching. Several crystals were selected for xenon exposure according to the above protocol and the best diffracting crystal was recorded at the ESRF, on beamline ID29. We optimally selected a wavelength of $1.77 \AA$ as to maximize the anomalous signal of xenon (the $\Delta \mathrm{f}^{\prime \prime}$ contribution is estimated greater than 9 electrons) and limit absorption of X-rays that becomes at low energy a serious limiting factor on intensities (and hence on data accuracy). The detector in use was a Pilatus3-6M (Dectris). The two OprN-wt and OprN-Xe data sets were indexed and merged with the XDS program [24] in the I4 tetragonal space group.

Data sets were scaled and merged with the POINTLESS/AIMLESS programs [25], part of the CCP4 suite $[26,27]$ and converted to structure factors with the CTRUNCATE program [28]. The two data sets showed low quality as deduced from their R factors agreement, especially in the case of OprN-Xe crystals because of low I/s(I) ratios resulting from the high absorption of diffracting spots at the selected wavelength. In that latter case, a full sphere of diffraction was recorded with a high redundancy with the aim to minimize errors by averaging the weak signals. This strategy was strengthened by Rpim values being always and systematically low, promising a fruitful exploitation of the diffraction data. The statistics of the best data collections are reported in Table 1 for the two OprN-wt and OprN-Xe structures. 
Table 1. Data recording statistics and refinements $(T=100 \mathrm{~K})$ for the OprN-wt and the OprN-Xe complex. Both are isomorphous, in the tetragonal 14 space group (Data from the highest resolution bin are in parentheses).

\begin{tabular}{|c|c|c|}
\hline & OprN-wt & OprN-Xe \\
\hline \multicolumn{3}{|c|}{ Data collection and integration statistics } \\
\hline Synchrotron beam line & ID23-2 & ID29 \\
\hline Wavelength $(\AA)$ & 0.873 & 1.771 \\
\hline Cell parameters $(\AA) a=b ; c$ & $257.65 ; 81.51$ & $256.41 ; 81.39$ \\
\hline Matthews coefficient $\left(\AA^{3} / \mathrm{Da}\right)$ & 2.32 & 2.29 \\
\hline Percentage of solvent (\%) & 47.04 & 46.36 \\
\hline \multirow[t]{2}{*}{ Resolution limits $(\AA)$} & $182.2-2.29$ & $181.0-2.50$ \\
\hline & $(2.34-2.29)$ & $(2.60-2.50)$ \\
\hline No of measured reflections & 584998 & 612875 \\
\hline No of unique reflections & 119774 & 91716 \\
\hline No of observed reflections & 113747 & 86977 \\
\hline Rmerge $(\%)^{a}$ & $13.3(67.0)$ & $19.2(67.2)$ \\
\hline $\operatorname{Rpim}(\%)^{a}$ & $7.3(42.0)$ & $7.4(32.0)$ \\
\hline $\mathrm{CC} 1 / 2(\%)^{\mathrm{b}}$ & $98.9(73.2)$ & $97.7(80.9)$ \\
\hline Completeness (\%) & $97.6(96.9)$ & $99.5(98.2)$ \\
\hline Redundancy & $4.9(4.5)$ & $6.7(6.2)$ \\
\hline $\mathrm{I} / \sigma(\mathrm{I})$ & $8.01(1.76)$ & $4.50(1.42)$ \\
\hline \multicolumn{3}{|l|}{ Refinement statistics } \\
\hline $\mathrm{R}_{\text {work }} / \mathrm{R}_{\text {free }}(\% / \%)$ & $18.55 / 22.53$ & $19.45 / 23.80$ \\
\hline No of used reflections & 113747 & 86977 \\
\hline No of refined atoms & 11430 & 10974 \\
\hline No of amino-acids & 1341 & 1341 \\
\hline No of water molecules & 684 & 550 \\
\hline No of ions & 2 & 2 \\
\hline No of alternates positions & 11 & 10 \\
\hline \multirow[t]{2}{*}{ No of additives } & 3 detergents & 4 detergents \\
\hline & 1 formate & 17 xenons \\
\hline rms bonds deviation $(\AA)$ & 0.02 & 0.02 \\
\hline rms angle deviation $\left({ }^{\circ}\right)$ & 2.074 & 2.046 \\
\hline Overall thermal factor $\left(\AA^{2}\right)$ & 35.64 & 31.09 \\
\hline \multicolumn{3}{|l|}{ Average thermal B factors } \\
\hline Main chain A / all atoms & $32.92 / 36.15$ & $29.14 / 31.20$ \\
\hline Main chain B / all atoms & $31.86 / 35.2$ & $28.45 / 30.48$ \\
\hline Main chain $\mathrm{C}$ / all atoms & $32.61 / 35.40$ & $29.57 / 31.19$ \\
\hline \multicolumn{3}{|l|}{ Ramachandran diagram } \\
\hline Preferred (\%) & 98.07 & 97.72 \\
\hline allowed (\%) & 1.78 & 1.60 \\
\hline poor (\%) & 0.15 & 0.68 \\
\hline PDB accession number & 5iuy & $5 n s w$ \\
\hline
\end{tabular}

${ }^{a}$ Rmerge is the standard agreement factor $=\sum_{H} \sum_{j}\left(\left|I_{H, j}-<I_{H}>\right|\right) / \sum_{H} \Sigma_{j}\left(I_{H, j}\right)$ and Rpim is the precisionindicating merging R-factor $=\sum_{H} \sqrt{ }\left(1 /(n-1) \sum_{j}\left(\left|I_{H, j}-<I_{H}>\right|\right) / \sum_{H} \sum_{j}\left(I_{H, j}\right)\right.$. The Rmerge of OprN-Xe was always rather high but trusting on the Rpim was always the guideline for data quality.

${ }^{b}$ from XDS statistics

https://doi.org/10.1371/journal.pone.0184045.t001 


\section{Structure determinations}

Initial phases of the OprN-wt structure were determined by molecular replacement (BALBES pipeline, [29]) using the $3 \mathrm{~d} 5 \mathrm{k}$ OprM structure from the PDB [13] as the starting model. The OprN-wt model was then reconstructed and refined independently of the 5azp OprN model deposited in the meantime at the PDB [17]. In the OprN-Xe structure, the xenon atoms were located from anomalous difference Fourier maps $\Delta \Delta \rho_{\text {an }}$ calculated with coefficients: $\Delta_{\text {ano }}^{ \pm}(H) . \exp \left[i\left(\phi(H)-\frac{\pi}{2}\right)\right]$ where $\Delta_{\text {ano }}^{ \pm}(H)$ are Bijvoet pair differences of structure factor moduli of OprN-Xe and $\phi(H)$ the phases of the refined OprN-wt structure.

\section{Structure refinements}

Refinements of both the native OprN-wt and the xenon complex OprN-Xe structures were performed using the REFMAC5 program [30,31] with individual isotropic factors. Standard constraints were used throughout on distances, angles, planes and isotropic factors. Refined models were checked using the Coot graphic program [32] for rebuilding's and water/ligands localization. In the OprN-Xe structure, special care was taken to correctly assign xenon occupancy factors as they are strongly correlated with their thermal factors within the refinement steps. After each round of REFMAC5 refinement, an omit-map was calculated with i) xenon atoms plus one sulphur atom of a methionine in each subunit (used as internal reference) subtracted from the model, and ii) the refined phases. Xenon occupancies were then calibrated with the omitted sulfur atoms, and a new round of REFMAC5 refinement was restarted. After two cycles of refinements/omit-map, the occupancy factors were found at a reasonably stable level and definitively assigned. In the last round of refinements, the thermal factors of xenon atoms were allowed to refine freely (not constrained).

All the statistics regarding the refined models are also reported in Table 1. The coordinates and structure factors of OprN-wt and OprN-Xe were deposited in the PDB (accession numbers 5iuy and 5nsw, respectively). All figures were drawn with Pymol [33]. Distances between Xenon and amino acid's bound atoms are in the range of 3.6-4.7 $\AA$, the complete list of the xenon sites and distances are reported in the Supporting Information (S1 and S2 Tables).

\section{Energy profile analyses}

A Fortran program was written to approximate the non-bonded energy of a xenon probe rolling along the central axis of the tunnel. The Lennard-Jones parameters and formulation of the non-bonded energy were taken from the CHARMM 22 forcefield for the $\mathrm{C}, \mathrm{N}, \mathrm{O}$, and $\mathrm{S}$ atoms [34]. Those of xenon were derived from literature, and combined Xe-C, Xe-O, Xe-N, and Xe-S interaction parameters were obtained from the Lorentz-Berthelot combining rules. A cut-off of $7 \AA$ was applied to the xenon atom interactions.

\section{Results}

\section{Description and comparison of the OprN structures}

As a representative member of the OMF family, OprN structure shows two main domains: a channel of $100 \AA$ long located in the periplasm, also known as the $\alpha$-domain, and a $\beta$-barrel of $30 \AA$ A long inserted in the outer membrane. The assembly of three monomers results in a long and wide tunnel responsible for the transport of drug out of the bacteria. In addition, an equatorial domain called the buoy domain, mainly composed of $\alpha$-helices and flexible loops is located in the middle of the $\alpha$-domain (Fig 1B). Surprisingly, this buoy domain is the main structural feature that differs among the OMFs proteins, both in structure and sequence. For instance, there is $31 \%$ of overall sequence identity between OprM and OprN, but only $23 \%$ for 
the buoy domain (structural alignments of OMFs are given in S1 Fig. An internal diameter of $35 \AA$ is measured along the channel between the b-barrel and the buoy. The protein channel is closed by a lid of three $\beta$-turns at the extracellular side and occluded by a constriction of three a-coiled-coils at the periplasmic side, which gating mechanism is probably ruled by an iris-like movement [14].

OprN-wt and OprN-Xe structures perfectly match each other with a C $\alpha$ rms deviation of 0.21 Å over 1352 atoms of the trimer, suggesting an overall protein fold unaffected by the xenon interaction. At the time we solved our OprN structure, another one appeared in the literature [17] solved in the same space group I4 (PDB code 5azp). The C $\alpha$ rms deviation between 5azp and our structure is about $0.77 \AA$. The main differences are limited to the C-terminus orientation as well as the distribution of the detergent and additive molecules around the trimer. In 5azp, all the $\beta$-octyl glucoside ( $\beta$-OG) molecules gather around the $\beta$-barrel domain, whereas in our OprN-wt structure, three $\beta$-OG were found at the $\beta$-barrel domain and one just above the buoy domain. The latter is trapped between the C-terminus, the $\alpha$-helices $\mathrm{H} 3$ and $\mathrm{H} 7$ of one subunit, and the a-coiled-coil tips of another subunit (Fig 1C and S2 Fig). Although this $\beta$-OG suggests a new binding site for a glucose derivative molecule on the channel of OprN, the crystal packing may have contributed to the stabilisation of this interaction (S2 Fig).

At the $\mathrm{N}$-terminus Cys residue, it was possible to construct at each monomer the acyl group of the post-translational palmitoylation. Although difficult to observe due to some disorder, 4 to 10 carbons of the aliphatic chains of the different palmitoyl were built. We also observed a dual acylation at the same modified cysteine, an unusual post-translational lipidation in the OMF family. In addition, a nickel cation complexed by the His 449 of the His-tag, Glu 139, and two water molecules, was located in the electron density map (S2A Fig).

\section{The xenon binding sites}

According to the anomalous map (Fig 2A), 18 peaks were located in OprN-Xe electron density. Among these peaks, 17 were identified as xenon atoms, according to their high hydrophobic environment, and one was attributed to an opportunistic nickel cation coordinated by the His-tag in subunit C (S2A Fig). Regarding xenon atoms, only one site is fully occupied, another shows a very faint signature ( 10\% occupancy). All the other xenon sites display partial occupancies in the range $20-40 \%$.

\section{The xenon binding sites in the $\alpha$-domain}

The main feature of the OprN-Xe structure is a site of xenon with $100 \%$ occupancy, named M1. This site is located on the non-crystallographic three-fold axis running along the channel at the periplasmic extremity of OprN. The xenon is trapped in sandwich between the three symmetry-related Leu 405 forming a tight hydrophobic constriction of the channel at that extremity (Fig 2C). The xenon cavity is lined on the other side by the three symmetry-related Asp 409, part of a key circular salt bridge with Arg 412, two residues also highly conserved in OprM (Asp 416-Arg 419, respectively).

Among the other sites, 8 are localized in the $\alpha$-domain and the buoy domain in three equivalent cavities of each monomer, thus accounting for three sites termed S1 to S3 (Fig 2). In the last S3 site, only two xenon atoms are clearly visible in the anomalous map, with intensities greater than $4 \sigma$. The third position corresponds to a small peak, at the level of the map average signal $(<1 \sigma)$. During the refinement steps, this signal was seriously degraded and further not observed in 2Fo-Fc electron density maps calculated with the refined phases (these maps are represented in S3-S5 Figs of the Supporting information). This site was thus considered void 

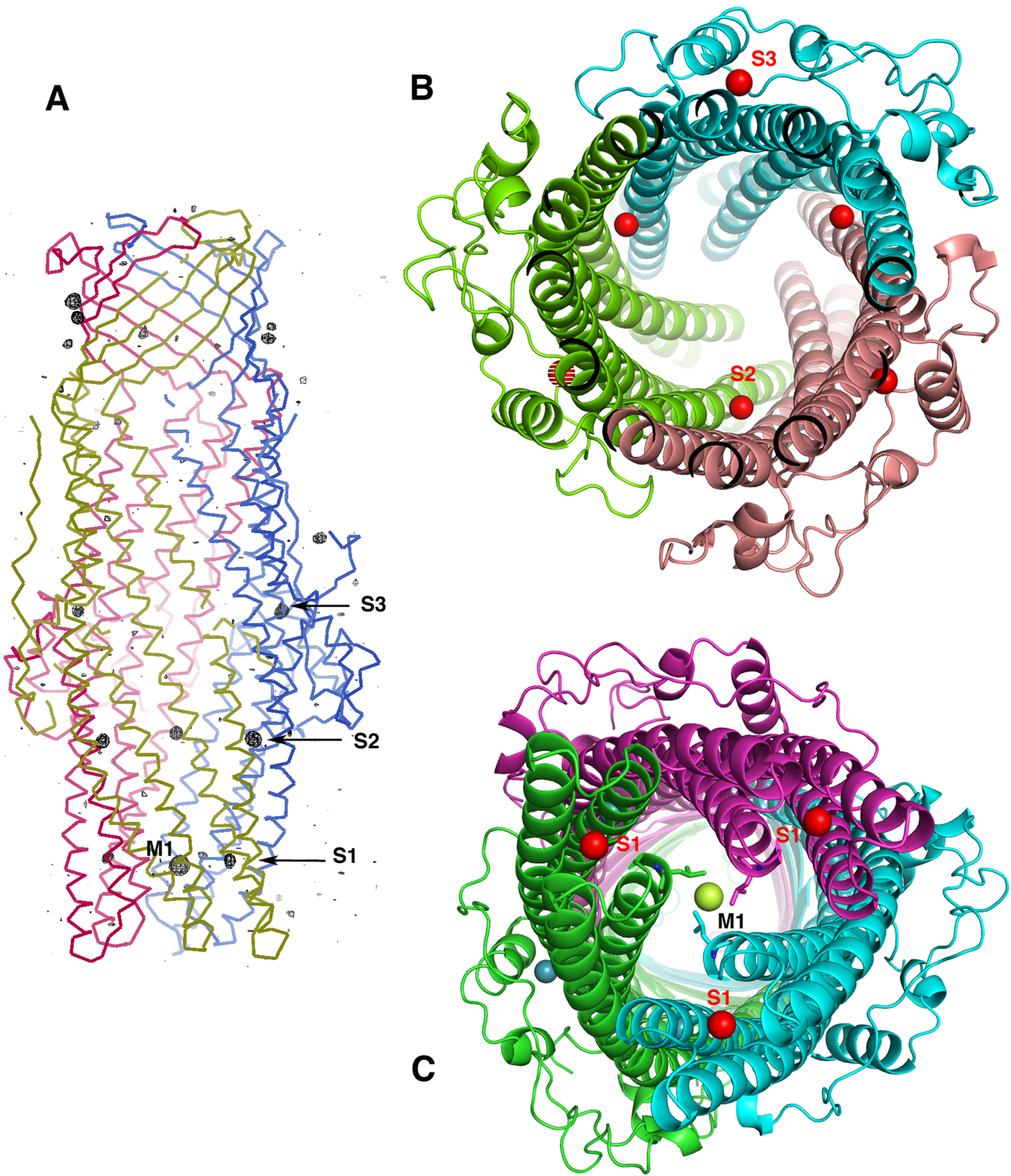

Fig 2. A: The anomalous map_-in black tracing and contour at $3.5 \sigma$._-superimposed to the Ca tracing of the OprN structure. The three S1 to S3 sites are labelled. On top of the structure, outside of the $\beta$-barrel, four more xenon atoms are present around the $\beta$-barrel domain, in a fully hydrophobic environment. B: (view from the extra cellular side) The two inter and intra-subunit S2 and S3 sites in the helical $\alpha$-domain (xenon as red spheres) viewed along the central channel of the trimer. C: (view from the periplasmic side) The M1 site locked by the three equivalent Leu 405 , viewed along the central channel of OprN-Xe structure at the periplasmic entrance. The S1 secondary sites are located at the same level of the main M1 site in a plane perpendicular to the tunnel. S1 xenon atoms are in red, M1 xenon in lime green. The three monomers building the cylindrical scaffold are in three different colours.

https://doi.org/10.1371/journal.pone.0184045.g002 
of xenon. The secondary sites $\mathrm{S} 1$ to $\mathrm{S} 3$ are all within the $\alpha$-domain, either intra-subunit (S1, S3) or inter-subunit (S2). In the first S1 site, three xenon atoms are located, at the vicinity of site M1 (Fig 2C) in a specific pocket with equivalent environment and built at the core of three of the four helices bundle in each monomer (Fig 2C). At the exception of Glu 411, presenting an oxygen atom at close distance of each xenon (although larger than $3.1 \AA$ ), the environment of S1 remains as expected mostly hydrophobic, with contacts to Leu 205, Leu 408, Phe 404, together with aliphatic side chains of Ser 180, Arg 181, Arg 204 and Gln 177 (Fig 3).

The secondary site S2 also includes three xenon atoms in a similar hydrophobic environment. It is located right below the buoy, at the interface of two subunits on the inner rim of the central tunnel. The three xenon atoms are caged by the aliphatic part of the Glu 427, Arg 375, Lys 371 side chains, from one subunit, and Gln 214, Pro 213, Ala 217 from another one (Fig 2B).

The last xenon atoms (sites S3) are located on the external face of the channel, above the buoy. They appear at the level of the junction between the two helices $\mathrm{H} 2$ and $\mathrm{H} 4$ parallel to the long one, H3, which extends along the whole periplasmic domain, in the most non-symmetric part of the trimer assembly. The first xenon ( $\mathrm{S} 3 \mathrm{c}$ site) is close to the $\beta-\mathrm{OG}$ molecule in the vicinity of the C-monomer His-tag (S2 Fig). The second xenon (S3b site) lies at the same position in molecule $\mathrm{B}$, despite the absence of $\beta$-OG in this monomer. The third xenon (S3a site) is only present at a low occupancy, probably less than $5 \%$, as deduced from its weak anomalous signature. This nearly void site could be attributed to the absence of a neighbour OprN molecule in the packing, and thus facing the four-fold axis of the tetragonal space group and the huge cavity built around that axis. In the two former S3 sites (S3a and S3b), the Leu 149 lateral chain is rotated out of its position as compared to the native OprN-wt structure, a feature rarely observed in xenon cavities, which always show equivalent side-chain orientations, with or without xenon.

Finally, a last xenon atom was observed in a cavity carved in the inner bank of the $\alpha$ domain, at the level of the buoy domain, in a hydrophobic zone of the tunnel (see Fig $2 \mathrm{~A}$ and S5 Fig).

\section{The xenon binding sites in the $\beta$-barrel domain}

Seven more xenon atoms are observed around the membrane $\beta$-barrel in the highly hydrophobic zone corresponding to the outer membrane bilayer (Fig 2A). These xenon atoms form a chain of discrete well-characterized sites, as observed in the S4 Fig, together with $2 \mathrm{Fo}-\mathrm{Fc}$ electron density maps.

\section{Hydropathy profile of the tunnel}

As proteins/Xenon interactions are closely linked to hydrophobic notions, it appeared important to explore the hydrophobicity variation along the inner face of the tunnel. The hydropathy profile was visualized by the ChExVis program [35] (Fig 4B), and the non-bonded energy for a xenon was calculated by rolling the atom along the tunnel axis (Fig 4C).

Two hydrophobic regions of the tunnel are highlighted in the ChExVis diagram of the cavity. The first hydrophobic region is localized at the periplasmic extremity (the M1 xenon site previously described). This constricted region is built by a number of conserved residues arranged in specific polar/hydrophobic/polar rings, stacked on top of each other's. This arrangement induces a strong gradient of polarity along the central channel and on a very short distance. the iris-like closed structure corresponds to a minimum energy at the Leucine405 triplet, in sandwich between the two high energy barriers of Asp-409 / Arg-412 on one side, and Asp-403 / Arg-396 on the other side. It is worth noting that an equivalent profile is 

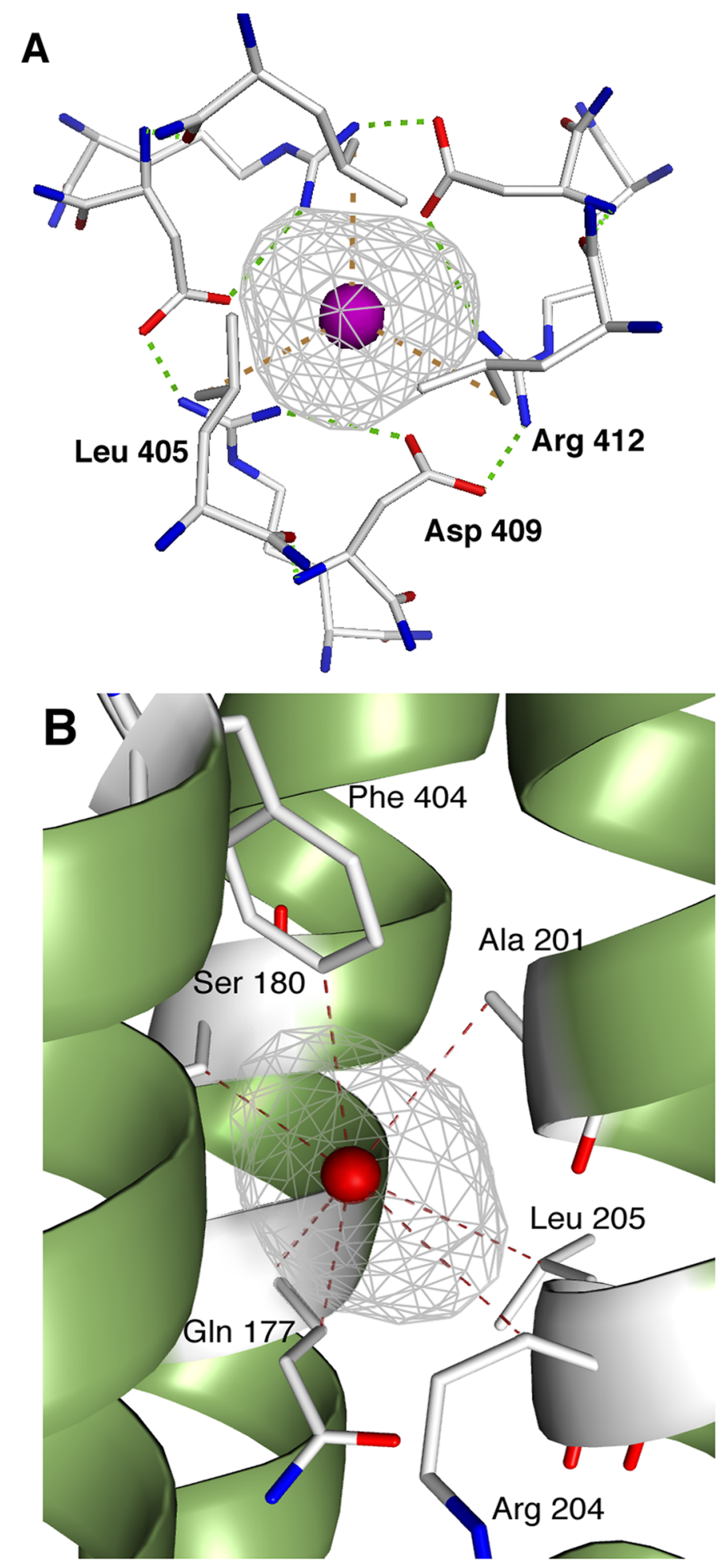

Fig 3. A: Details of the xenon's cavity M1 viewed along the tunnel. The cavity (in mesh) is built by the three hydrophobic Leu 405 side chains on one edge, and the circular salt bridge system [.. -> Asp 409a-Arg $412 b \rightarrow$ Asp 409b-Arg 412c $\rightarrow$ Asp 409c-Arg 412a $\rightarrow .$.$] on the other. Hydrophobic contacts to Leu are in$ brown, hydrogen bonds between Glu and Arg are in green. B: Residues building the site $S 1$, at the same level as M1. Note that the hydrophobic contacts towards polar residues like GIn or Arg, displace the polar part of their side chains as to keep the overall hydrophobic character of the cavity. Xenon atoms are represented as spheres with arbitrary radius.

https://doi.org/10.1371/journal.pone.0184045.g003 

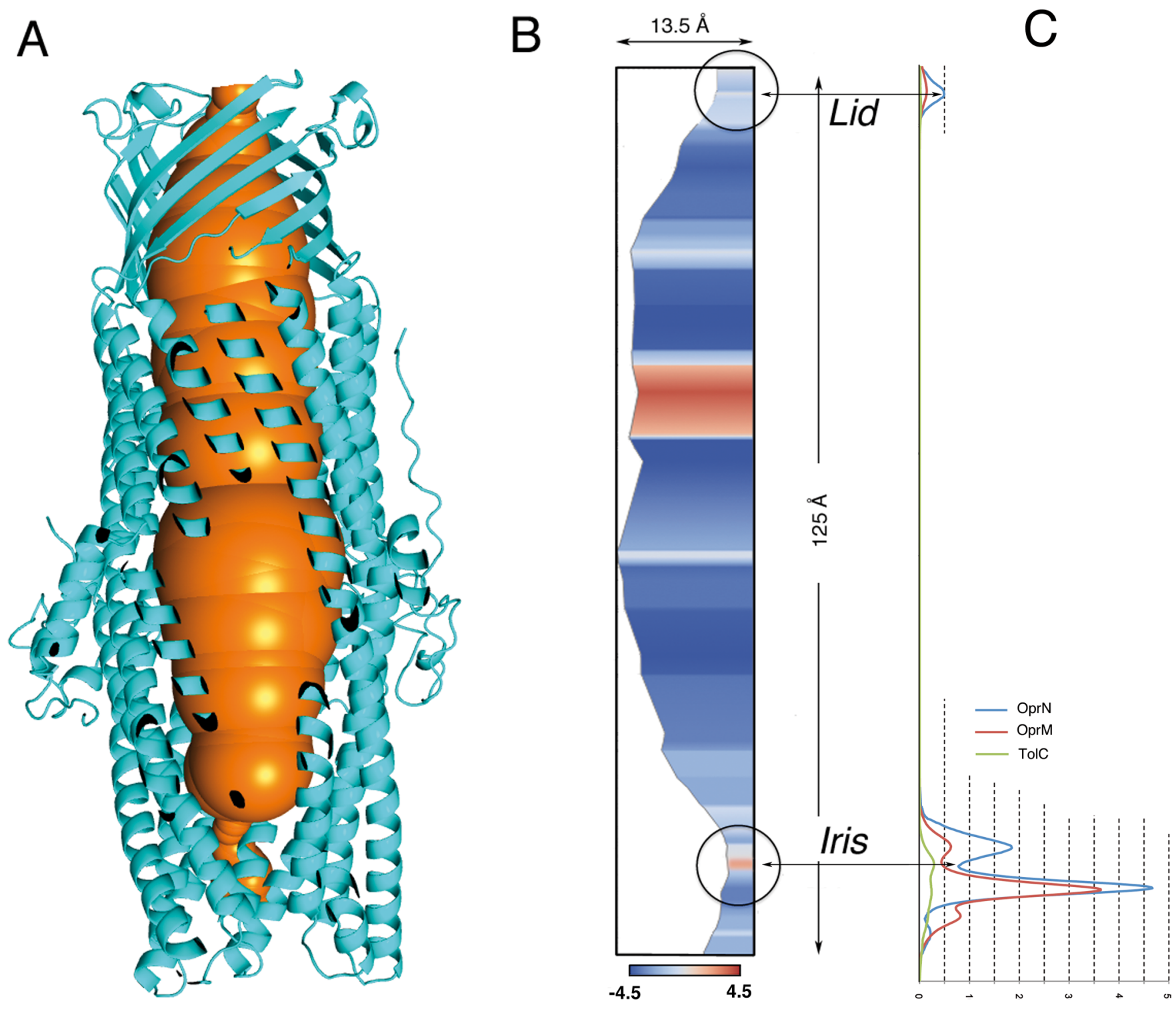

Non bonded Energy (kJ.mol-1) $\times 10$

Fig 4. A: The huge central cavity of OprN constricted at both ends, towards the periplasm (bottom), and the exterior of the cell (top). B: At the same scale, the cavity's profile as seen by the ChExVis program [35] with the two constricted regions pointed by the two red arrows. The gradient profile of the cavity is given as the standard Kyte \& Doolittle scale of polar/hydrophobic amino-acids (color code is blue to red for increasing hydropathy). C: Non-bonded energy calculated for a xenon atom rolling along the central tunnel of OprN, superposed to the same calculations with OprM and TolC structures (3d5K and 1ek9 from PDB). OprN, OprM, and TolC traces are in blue, orange and lime colors. Energies are calculated using the LennardJones parameters from the CHARMM22 forcefield [34].

https://doi.org/10.1371/journal.pone.0184045.g004

obtained from the OprM tunnel crossing but not for TolC, in which the channel entrance is wider.

The second and larger hydrophobic zone is located at the junction of the $\beta$-barrel domain and the periplasmic $\alpha$-domain and built by three hydrophobic residues, Leu 70, Val 74, and Leu 134, pointing inward the cavity. Associated three by three, they construct a cylinder about 18 to $20 \AA ̊$ in diameter and $8 \AA$ long leading to a sharp change in the overall polarity along the tunnel at this level. Equivalent hydropathy changes can also be observed in other OMF's like 
TolC or OprM but they are differently distributed along their tunnel axis, pointing out differences that could be associated to a selectivity for a given class of drugs, as experimentally observed [3, 8]. The role of this particular hydrophobic patch is not obvious; it should be implicated in triggering the concerted motions of the helical domains in the overall dynamic of the open/close motions leading to the drug efflux, a motion recently investigated by normal mode analyses on the homologous OprM [13]. A xenon site was observed at this level (Xe-507 in S2 Table, S5 Fig).

\section{Discussion}

Noble gases are known to be chemically non-reactive but biochemically active compounds. In particular, xenon has remarkable properties as inhalational clinical anaesthetic [36-38], together with strong neuroprotective properties $[39,40]$. Crystallographic studies under pressure have allowed a better understanding of the mechanisms by which these inert gases act toward a large panel of proteins, and especially neuronal targets, as demonstrated on protein models [41], and functional gated ion channels [22]. The remarkable and widely observed interactions of xenon with proteins are now clearly understood as a multi-process perturbation in their catalytic activities. Even if a small and reversible interaction may affect a single localized protein in a multi-component system or in signalling pathways, a large perturbation effect is expected at the end of the cascade after the sum of many individual weak interactions. On the other hand, hydrophobic inner cavities in proteins play key roles in their function [42, 43]. Their flexibility/rigidity allow to optimize the catalytic efficiency by lowering in energy landscapes the Gibbs free energy of their processes [44, 45]. Varying and playing with the thermodynamic parameters (Pressure and Temperature) of the processes is a way to tackle this problem $[46,47]$. Interactions of both xenon and cavities are far from being simple processes, and many studies were devoted to untangle these associations [48-50].

Xenon interacts mainly through induced-induced dipolar interactions (London forces, varying as $\sim 1 / \mathrm{r}^{6}$ ) that become rather efficient at short distances when xenon nests in small cavities and develops many interactions toward hydrophobic amino acid side chains around without disturbing too much the overall conformation of the cavity [20]. As such, xenon cannot be considered as a true inhibitor but just as a "troublemaker" able to disturb functional dynamic properties of its target. Simply releasing xenon pressure restores the full activity. Such a behaviour was proposed from kinetic experiments with globular proteins under xenon atmosphere [49] and may be extended to the OprN-Xe complex.

It is now widely admitted that the gated function and the open/close equilibrium of OMF's, and especially OprN, is critically controlled by the two extremities of the $124 \AA$ long tunnel formed upon the molecular trimerization. The main gated region is the iris-like structure located in the periplasm and connected to the RND molecular transporter (Figs 1 and 4). This region corresponds exactly to the main site of xenon, M1, the most important feature observed here in the OprN-Xe structure. It shows uncommon aspects: purely hydrophobic on one side (the Leu 405 ring), and strongly polar on the other side (the circular ring of salt bridges Asp 409 / Arg 412). This region was also described to be responsible of maintaining the closed state of the channel OprM [13] (Figs 3A and 4B and 4C for OprN). Similarly, in TolC, the corresponding and conserved Asp 374 forms a ring that prevents the export when cross-linked [51]. In addition, Asp 374 associated with Asp 371, is also responsible of the cobalt hexamine blockade of the TolC channel [16].

The specific topology of the M1 site, with a thin hydrophobic zone in sandwich between two polar regions, can be considered as an additional driving force expelling the drug out of the cell to the passive efflux mechanism. 
Regarding the secondary sites, all located between intra or inter coiled-coil helices responsible of the concerted open/close allostery, they are likely to rigidify the overall coiled-coil structure of the $\alpha$-domain by introducing additional constrictions when occupied by xenon atoms, a feature previously observed on globular proteins [50]. Here again, because of the lack of an OprN open-state X-ray structure for comparisons, it is difficult to firmly assess this hypothesis.

In addition to the $\mathrm{M}$ and $\mathrm{S}$ sites all located in the a-domain, seven additional xenon atoms are observed at the level of the $\beta$-barrel domain (Fig $3 \mathrm{~A}$ ). They interact directly with the $\beta$ strands of the three subunits building the 12-stranded $\beta$-barrel locked in the outer membrane, filling hydrophobic cavities built around the $\beta$-barrel domain. These sites are indeed not symmetric as compared to the others, and are likely to have no biological significance. They however protect and stabilize the $\beta$-barrel of OprN against the external polar medium, a feature already observed in porin's crystals under xenon pressure [52].

\section{Conclusion}

The OprN channel of the drug transporter MexEF-OprN was analysed in the presence of xenon by X-ray diffraction. The present study evidences the key role of the central iris-like bottleneck built by the highly conserved residues Leu 405 on one side, and Asp 409, Arg 412, on the other side, all forming the molecular closing system suspected to control the open/close state of the channel in the periplasm. Xenon highlights a strong discontinuity in the hydrophobic gradient along the channel at this level that might be an additional driving force to the passive mechanism for the drug transport out of the cell. However, this important cavity shows a higher polarity and is known to host cobalt hexamine, characterized as an opening blocker in TolC, another member of the family. Even if xenon cannot be considered as an inhibitor, it is likely to hamper in the specific case of OMF's the two proposed transport mechanisms: the iris-like mechanism on the periplasmic side, by filling this constricted key-cavity along the channel, and the overall concerted stretch/rotation mechanism by filling specific hydrophobic cavities, built by the coiled-coil long helices forming the cylindrical scaffold of the $\alpha$-domain.

\section{Supporting information}

S1 Table. The xenon sites in OprN- $\alpha$ domain (at 20 bar).

\section{S2 Table. The xenon sites in $\mathrm{OprN}-\beta$ barrel domain (at 20 bar).} (PDF)

S1 Fig. Structural alignment of different OMFs with OprN. According to ENDscript (Robert, X., Gouet, P., Nucl. Acids. Res. (2014) 42(w1): w320-324). Pdb codes 5IUY, 3DK5, 5AZS, 4MT0, 4MT4, 3PIK and 1YC9 correspond to OMF proteins OprN, OprM, OprJ, MtrE, CmeC, $\mathrm{CusC}$, and $\mathrm{VceC}$, respectively. The amino acid sequence numbering corresponds to OprN. Strictly identical residues are highlighted in red and similar residues in yellow. The buoy domain is represented by the blue boxes. "acc" is the relative residue accessibility: buried in white, intermediate in cyan, and accessible in blue. "hyd" is the hydropathy scale, from hydrophobic in pink to intermediate in grey, and hydrophilic in cyan. (PDF)

S2 Fig. A: The His-tag on one monomer at left (blue $\mathrm{C}$ chain), the $\beta$-OG molecule (yellow $\mathrm{C}$ chain), the nickel atom (green sphere) and a xenon (S3 site-pink sphere), are shown. They are observed parallel to the 136-146 helix in the buoy domain, and are responsible of breaking the three-fold symmetry at this level of the trimer. B: Crystal packing of OprN in the I4 space group showing the perpendicular interaction of two channels responsible of the non- 
equivalence observed at site S3.

(PDF)

S3 Fig. OprN xenon sites. The 2Fo-Fc maps are drawn at electron density greater than $2.5 \sigma$ the map average. (A: site M1, B: one of the S1 sites at the same level). C and D: The two secondary S2 and S3 sites in subunit B.

(PDF)

S4 Fig. Three xenon sites lining the periphery of the hydrophobic $\beta$-barrel domain. The 2Fo-Fc map is contoured at $2.5 \sigma$. Top: site Xe 503B, lower left: Xe 502B and lower right: Xe 501B (see S2 Table).

(PDF)

S5 Fig. The xenon atom located at the level of the buoy domain. Located in the wide hydrophobic patch of the tunnel. $(\mathrm{PDF})$

\section{Acknowledgments}

We acknowledge the ESRF for the use of their synchrotron radiation facilities and thank the staff at the ID29 and ID23-2, beamlines for help and advices.

\section{Author Contributions}

Conceptualization: Arnaud Ducruix, Thierry Prangé.

Data curation: Gilles Phan, Thierry Prangé.

Formal analysis: Gilles Phan, Thierry Prangé, Isabelle Broutin.

Funding acquisition: Isabelle Broutin.

Investigation: Yvette Véronique Ntsogo Enguéné, Gilles Phan, Cyril Garnier, Thierry Prangé, Isabelle Broutin.

Methodology: Thierry Prangé.

Project administration: Isabelle Broutin.

Resources: Isabelle Broutin.

Software: Gilles Phan, Thierry Prangé, Isabelle Broutin.

Supervision: Arnaud Ducruix, Thierry Prangé, Isabelle Broutin.

Validation: Gilles Phan, Cyril Garnier, Arnaud Ducruix, Thierry Prangé, Isabelle Broutin.

Visualization: Yvette Véronique Ntsogo Enguéné, Gilles Phan, Arnaud Ducruix, Thierry Prangé.

Writing - original draft: Gilles Phan, Arnaud Ducruix, Thierry Prangé, Isabelle Broutin.

Writing - review \& editing: Arnaud Ducruix, Thierry Prangé.

\section{References}

1. Lister PD, Wolter DJ, Hanson ND. Antibacterial-resistant Pseudomonas aeruginosa: Clinical impact and complex regulation of chromosomally encoded resistance mechanisms. Clin Microbiol Rev. 2009; 22:582-610. https://doi.org/10.1128/CMR.00040-09 PMID: 19822890 
2. Puzari M, Chetia P. RND efflux pump mediated antibiotic resistance in Gram-negative bacteria Escherichia coli and Pseudomonas aeruginosa: a major issue worldwide. World J Microbiol Biotechnol. 2017; 33:24. https://doi.org/10.1007/s11274-016-2190-5 PMID: 28044273

3. Schweizer HP. Efflux as a mechanism of resistance to antimicrobials in Pseudomonas aeruginosa and related bacteria: unanswered questions. Genet Mol Res. 2003; 2:48-62. PMID: 12917802

4. Andersen C. Channel-tunnels: outer membrane components of type I secretion systems and multidrug efflux pumps of Gram-negative bacteria. Rev Physiol Biochem Pharmacol. 2003; 147:122-65. https:// doi.org/10.1007/s10254-003-0008-y PMID: 12783268

5. Stover CK, Pham XQ, Erwin AL, Mizoguchi SD, Warener P, Hickey MJ, et al. Complete genome sequence of Pseudomonas aeruginosa PA01, an opportunistic pathogen. Nature. 2000; 406:959-64. https://doi.org/10.1038/35023079 PMID: 10984043

6. Nakajima A, Sugimoto Y, Yoneyama H, Nakae T. High-level fluoroquinone resistance in Pseudomonas aeruginosa due to interplay of MexAB-OprM efflux pump and the DNA gyrase mutation. Microbiol Immunol. 2002; 46:391-5. PMID: 12153116

7. Nakajima A, Sugimoto Y, Yoneyama H, Nakae T. Localization of the outer membrane subunit OprM of resistance-nodulation-cell division family multicomponent efflux pump in Pseudomonas aeruginosa. $J$ Biol Chem. 2000; 275:30064-8. https://doi.org/10.1074/jbc.M005742200 PMID: 10889211

8. Phan G, Picard M, Broutin I. Focus on the outer membrane factor OprM, the forgotten player from efflux pumps assemblies. Antibiotics. 2015; 4:544-66. https://doi.org/10.3390/antibiotics4040544 PMID: 27025640

9. Du D, Wang Z, James NR, Voss JE, Klimont E, Ohene-Agyei T, et al. Structure of the AcrAB-TolC multidrug efflux pump. Nature. 2014; 509:512-5. https://doi.org/10.1038/nature13205 PMID: 24747401

10. Lambert O, Benabdelhak H, Chami M, Jouan L, Nouaille E, Ducruix A, et al. Trimeric structure of OprN and OprM efflux proteins from Pseudomonas aeruginosa, by 2D electron crystallography. J Struct Biol. 2005; 150:50-7. https://doi.org/10.1016/j.jsb.2005.01.001 PMID: 15797729

11. Wong KK, Hancock RE. Insertion mutagenesis and membrane topology model of the Pseudomonas aeruginosa outer membrane protein OprM. J Bacteriol. 2000; 182:2402-10. PMID: 10762238

12. Akama $H$, Kanemaki $M$, Yoshimura $M$, Tsukihara $T$, Kashiwagi $T$, Yoneyama $H$, et al. Crystal structure of the drug discharge outer membrane protein, OprM of Pseudomonas aeruginosa: dual modes of membrane anchoring and occluded cavity end. J Biol Chem. 2004; 279:52816-9. Epub 52816. https:// doi.org/10.1074/jbc.C400445200 PMID: 15507433

13. Phan G, Benabdelhak H, Lascombe MB, Benas $P$, Rety S, Picard M, et al. Structural and dynamical insights into the opening mechanism of $P$. aeruginosa OprM channel. Structure. 2010; 18:507-17. https://doi.org/10.1016/j.str.2010.01.018 PMID: 20399187

14. Koronakis V, Sharff A, Koronakis E, Luisi BF, Hughes C. Crystal structure of the bacterial membrane protein TolC central to multidrug efflux and protein export. Nature. 2000; 405:914-9. https://doi.org/10. 1038/35016007 PMID: 10879525

15. Koch DC, Raunest M, Harder T, Kandt C. Unilateral access regulation: Ground state dynamics of the Pseudomonas aeruginosa outer membrane efflux duct OprM. Biochemistry. 2013; 52:178-87. https:// doi.org/10.1021/bi3014714 PMID: 23234291

16. Higgins M, Eswaran J, Edwards P, Schertler G, Hughes C, Koronakis V. Structure of the ligand-blocked periplasmic entrance of the bacterial multidrug efflux protein TolC. J Mol Biol. 2004; 342:697-702. https://doi.org/10.1016/j.jmb.2004.07.088 PMID: 15342230

17. Yoneyhara R, Yamashita E, Nakagawa A. Crystal structures of OprN and OprJ, outermembrane factors of multidrug tripartite efflux pumps of Pseudomona aeruginosa. Proteins. 2016; 84:759-69. https://doi. org/10.1002/prot.25022 PMID: 26914226

18. Broutin I, Benabdelhak H, Moreel X, Lascombe MB, Lerouge D, Ducruix A. Expression, purification, crystallization and preliminary X-ray studies of the outer membrane efflux proteins OprM and OprN from Pseudomonas aeruginosa. Acta Cryst. 2005; F61:315-8.

19. Prangé $T$, Schiltz M, Pernot L, Colloc'h N, Longhi S, Bourguet W, et al. Exploring hydrophobic sites in proteins with xenon or krypton. Proteins. 1998; 30:61-73. PMID: 9443341

20. Schiltz M, Fourme R, Prangé T. Use of noble gases xenon and krypton as heavy atoms in protein structure determination. Meth Enzymol. 2003; 374:83-119. https://doi.org/10.1016/S0076-6879(03)74004-X PMID: 14696369

21. Hayakawa N, Kasahara T, Hasegawa D, Yoshimura K, Murakami M, Kouyama T. Effect of xenon binding to a hydrophobic cavity on the proton pumping cycle in bacteriorhodopsin. J Mol Biol. 2008; 384 (4):812-23. https://doi.org/10.1016/j.jmb.2008.09.075 PMID: 18930734 
22. Sauguet L, Fourati Z, Prangé $T$, Delarue M, Colloc'h N. Structural Basis for Xenon Inhibition in a Cationic Pentameric Ligand-Gated Ion Channel. PloS One. 2016; 11:e0149795. https://doi.org/10.1371/ journal.pone.0149795 PMID: 26910105

23. Winter MB, Herzik MAJ, Kuriyan J, Marletta MA. Tunnels modulate ligand flux in a heme nitric oxide/ oxygen binding (H-NOX) domain. Proc Natl Acad Sci USA. 2011; 108:E881,E9. https://doi.org/10.1073/ pnas. 1114038108 PMID: 21997213

24. Kabsch W. XDS. Acta Cryst. 2010; D66:125-32.

25. Evans PR. Scaling and assessment of data quality. Acta Cryst. 2006; D62:72-82.

26. CCP4. The CCP4 suite of programs for protein crystallography: Collaborative Computational Project 4. Acta Cryst. 1994; D50:760-3.

27. Winn MD, Ballard CC, Cowtan K, Dodson EJ, Emsley P, Evans PR, et al. Overview of the CCP4 suite and current developments. Acta Cryst. 2011; D67:235-42.

28. French GS, Wilson KS. On the treatment of negative intensity observations. Acta Cryst. 1978; A34:517-25.

29. Long F, Vagin A, Young P, Murshudov GN. BALBES: a molecular-replacement pipeline. Acta Cryst. 2008; D64:125-32.

30. Murshudov GN, Vagin AA, Dodson EJ. Refinement of macromolecular structures by the MaximumLikelihood method. Acta Cryst. 1997; D53:240-55.

31. Murshudov GN, Skubák P, Lebedev AA, Pannu NS, Steiner RA, Nicholls RA, et al. REFMAC5 for the refinement of macromolecular crystal structures. Acta Cryst. 2011; D67:355-67.

32. Emsley P, Cowtan K. Coot: Model-Building Tools for Molecular Graphics. Acta Cryst. 2004; D60:212632.

33. DeLano WL. The PyMOL Molecular Graphics System. DeLano Scientific LLC, Palo Alto, California, USA, 2002.

34. MacKerell JAD, Bashford D, Bellott M, Dunbrack RL Jr., Evanseck JD, Field MJ, et al. All-atom empirical potential for molecular modeling and dynamics Studies of proteins. J Phys Chem. 1998; B102:3586616.

35. Masood TB, Sandhya S, Chandra N, Natarajan V. CHEXVIS: a tool for molecular channel extraction and visualization. BMC Bioinformatics. 2015; 16(119):1-19.

36. Abraini JH, Marassio G, David HN, Vallone B, Prangé T, Colloc'h N. Crystallographic Studies with Xenon and Nitrous Oxide Provide Evidence for Protein-dependent Processes in the Mechanisms of General Anesthesia. Anaesthesiology. 2014; 121:1018-27.

37. Cullen SC, Gross EG. The anesthetic properties of xenon in human and animal beings, with additional observations on krypton. Science. 1951; 113:580-2. PMID: 14834873

38. Eckenhoff RG. Promiscuous ligands and attractive cavities: how do the inhaled anesthetics work? Molecular Interventions. 2001; 1:258-68. PMID: 14993365

39. David HN, Haelewyn B, Rouillon C, Lecoq M, Chazalviel L, Apiou G, et al. Neuroprotective effects of xenon: A therapeutic window of opportunity in rats subjected to transient cerebral ischemia. Faseb J. 2008; 22:1275-86. https://doi.org/10.1096/fj.07-9420com PMID: 18024836

40. Harris PD, Armstrong SP, Campos-Pires R, Kiru L, Franks NP, Dickinson R. Neuroprotection against traumatic brain injury by xenon, but not argon, is mediated by inhibition at the N-Methyl-D-Aspartate Receptor glycine site. Anaesthesiology. 2013; 119:1137-48.

41. Colloc'h N, Sopkova-de Olivera Santos J, Retailleau P, Vivarès $D$, Bonneté F, Langlois d'Estainto B, et al. Protein Crystallography under Xenon and Nitrous Oxide Pressure: Comparison with In Vivo Pharmacology Studies and Implications for the Mechanism of Inhaled Anesthetic Action. Biophys J. 2007; 92:217-24. https://doi.org/10.1529/biophysj.106.093807 PMID: 17028130

42. Hubbard SJ, Argos P. A functional role for protein cavities in domain: domain motions. J Mol Biol. 1996; 261:289-300. https://doi.org/10.1006/jmbi.1996.0460 PMID: 8757295

43. Hubbard SJ, Gross $\mathrm{KH}$, Argos P. Intramolecular cavities in globular proteins. Protein Engineering. 1994; 7:613-26. PMID: 8073031

44. Frauenfelder H, Sligar SG, Wolynes PG. The energy landscapes and motions of proteins. Science. 1991; 254:1598-603. PMID: 1749933

45. Frauenfelder $\mathrm{H}$, Fenimore $\mathrm{PW}$, Young RD. Protein dynamics and function: insights from the energy landscape and solvent slaving. IUBMB Life. 2007; 59:506-12. https://doi.org/10.1080/ 15216540701194113 PMID: 17701545

46. Girard E, Marchal S, Perez J, Finet S, Kahn R, Fourme R, et al. Structure-function perturbation and dissociation of tetrameric urate oxidase by high hydrostatic pressure. Biophys J. 2010; 98:2365-73. https://doi.org/10.1016/j.bpj.2010.01.058 PMID: 20483346 
47. Fourme R, Girard E, Kahn R, Dhaussy AC, Ascone I. Advances in high-pressure biophysics: status and prospects of macromolecular crystallography. Ann Rev Biophys. 2009; 38:153-71.

48. Quillin ML, Breyer WA, Griswold IJ, Matthews BW. Size versus polarizability in protein-ligand interactions: binding of noble gases within engineered cavities in phage T4 lysozyme. J Mol Biol. 2000; 302:955-77. https://doi.org/10.1006/jmbi.2000.4063 PMID: 10993735

49. Marassio G, Prangé T, David HN, Santos JS, Gabison L, Delcroix N, et al. Pressure-response analysis of anesthetic gases xenon and nitrous oxide on urate oxidase: a crystallographic study. Faseb J. 2011; 25(7):2266-75. https://doi.org/10.1096/fj.11-183046 PMID: 21421845

50. Colloc'h N, Prangé T. Functional relevance of the internal hydrophobic cavity of urate oxidase. FEBS Letters. 2014; 588:1715-9. https://doi.org/10.1016/j.febslet.2014.03.017 PMID: 24657440

51. Eswaran J, Hugues $C$, Koronakis V. Locking TolC entrance helices to prevent protein translocation by the bacterial type I export apparatus. J Mol Biol. 2003; 327:309-15. PMID: 12628238

52. Sauer $O$, Roth $M$, Schirmer T, Rummel G, Kratky C. Low-resolution detergent tracing in protein crystals using xenon or krypton to enhance X-ray contrast. Acta Cryst. 2002; D60:60-9. 